\title{
Left atrial mechanical function and stiffness in patients with atrial septal aneurysm: A speckle tracking study
}

\author{
Mehmet Demir, İbrahim Aktaş, Sencer Çamcı \\ Department of Cardiology, Bursa Yüksek İhtisas Education and Research Hospital, Bursa, Turkey
}

\begin{abstract}
Background: Atrial septal aneurysm (ASA) is a risk factor for arterial embolism. Atrial dysfunction and atrial arrhythmia, such as atrial fibrillation, might represent a mechanism for arterial embolism in such patients. Speckle tracking echocardiography (STE) is a novel and promising tool for detecting early changes in left atrial (LA) myocardial dysfunction. The aim of the study was to evaluate LA mechanical function and stiffness in ASA patients by 2-dimensional STE strain parameters.
\end{abstract}

Methods: Thirty-four ASA patients (44.2 \pm 12.3 years, 15 male) were studied, using a STE, and were compared with 31 age, gender, and left ventricular (LV) mass-matched controls (41.8 \pm \pm 11.5 years, 14 male). LA volume indices, mitral annular velocities, and global longitudinal $L A$ strain were measured. The ratio of E/e' to LA strain was used as an index of LA stiffness.

Results: Patients with ASA showed increased LA volume indices and decreased LA global strain (25.3 \pm 5.2 vs. $42.1 \pm 8.7, p<0.001)$. LA stiffness was increased in patients with ASA compared to the control subjects $(0.41 \pm 0.15$ vs. $0.14 \pm 0.05, p<0.001)$, and LA strain and stiffness were related with LA volume indices.

Conclusions: Patients with ASA have decreased LA global strain and increased stiffness, in comparison with those of the control subjects. LA strain and stiffness were significantly related with LA volume indices. LA stiffness and strain can be used for the assessment of $L A$ function in patients with ASA. (Cardiol J 2015; 22, 5: 535-540)

Key words: atrial septal aneurysm, atrial dysfunction, atrial stiffness, arrhythmia

\section{Introduction}

Atrial septal aneurysm (ASA) is a risk factor for arterial embolism, as it frequently co-exists with an atrial septal defect (ASD) or patent foramen ovale (PFO). The incidence of ASA, depending on the imaging method, is $0.2-4 \%$ transthoracic echocardiography (TTE) and 2-8\% transesophageal echocardiography (TEE). Despite prior reports concerning paradoxical embolism through a PFO, the magnitude of this phenomenon as a risk factor for a stroke remains undefined because deep venous thrombosis is infrequently detected in such patients. Atrial dysfunction and atrial arrhythmia, such as atrial fibrillation (AF), might represent an alternate and additional mechanism for arterial embolism in these patients [1-3].

Recently, the assessment of left atrium (LA) deformation profiles obtained by deformation imaging has been proposed as an alternative method of exploring LA function and to detect early changes in LA myocardial performance. Several studies

Address for correspondence: Mehmet Demir, MD, Bursa Yüksek İhtisas Education and Research Hospital, Bursa 16100, Turkey, tel: +90 2243605050, fax: +90 2243605055, e-mail: drmehmetmd@gmail.com 
have shown that LA stiffness increases with atrial remodeling and reflects a deteriorated reservoir function [4-6].

However, to date, little has been known with regard to the change of LA mechanical function, including LA stiffness, in patients with ASA.

Our aim was to evaluate LA mechanical function and stiffness in ASA patients by 2-dimensional (2D) speckle tracking echocardiography (STE) strain parameters.

\section{Methods}

\section{Selection of the patients}

Thirty-four patients with ASA that had been identified during echocardiographic evaluation performed for various reasons ( 15 male, 19 female; mean age $44.2 \pm 12.3$ years) were entered into the present study. Thirty-one age-matched adults (14 male, 17 female; mean age $41.8 \pm 11.5$ years) who had normal cardiac structures on echocardiography comprised the control group.

Entry criteria included the presence of ASA determined by echocardiography and sinus rhythm on surface electrocardiogram (ECG). A physical examination, medical history and blood biochemistry testing were conducted in both patient groups. The subjects were defined as hypertensive if their blood pressure was $\geq 140 / 90 \mathrm{~mm} \mathrm{Hg}$ or if they were receiving any antihypertensive medication. Diabetes mellitus was defined as a history of antidiabetic medication use or a fasting glucose level $>126 \mathrm{mg} / \mathrm{dL}$. Smoking status was classified as smokers and those who never smoked. Patients with coronary artery disease, heart failure, valve disease, cardiomyopathy, hypertension, diabetes mellitus, chronic lung disease, thyroid dysfunction, anemia, and renal and hepatic insufficiency were excluded from the study.

The study was approved by the local bioethical committee and all patients gave their informed consent.

\section{Echocardiographic measurements}

M-mode, 2D, pulsed and color-flow Doppler echocardiographic examinations of all the subjects were performed by the same examiner using a commercially available device (Philips IE33; Philips, The Netherlands) (equipped with a $1-5 \mathrm{MHz}$ phased-array transducer). During echocardiography, a single-lead ECG was recorded continuously.

ASA was detected by TTE. Patients who had an ASA with a base $>15 \mathrm{~mm}$ and protrusion $>10 \mathrm{~mm}$ were included in the study $[1,7]$.
LA and left ventricular (LV) end-systolic and end-diastolic diameters were measured. LV ejection fraction (LVEF) was estimated using Simpson's rule.

Pulsed-wave mitral and tricuspid flow velocities were measured from the apical 4-chamber view by inserting a sample volume to leaflet tips. Mitral and tricuspid early diastolic velocity $(\mathrm{E}, \mathrm{cm} / \mathrm{sn})$, late diastolic velocity $(\mathrm{A}, \mathrm{cm} / \mathrm{sn})$, and $\mathrm{E} / \mathrm{A}$ ratio were determined. Each representative value was obtained from the average of 3 measurements.

Tissue Doppler imaging echocardiography was performed by transducer frequencies of $3.5-4.0 \mathrm{mHz}$, adjusting the spectral pulsed Doppler signal filter until a Nyquist limit of $15-20 \mathrm{~cm} / \mathrm{sn}$ was reached, and using the minimal optimal gain. The monitor sweep speed was set at $50-100 \mathrm{~mm} / \mathrm{s}$ to optimize the spectral display of myocardial velocities. Myocardial peak systolic s', $\mathrm{cm} / \mathrm{s}$ ), early (e', $\mathrm{cm} / \mathrm{s})$, and late $\left(a^{\prime}, \mathrm{cm} / \mathrm{s}\right)$ diastolic velocities were obtained by placing a tissue Doppler sample volume in the basal segments of lateral and septal walls for the $\mathrm{LV}$. Each parameter was measured as the average of at least 3 consecutive beats.

From the apical 4- and 2-chamber view, the following LA volumes were measured using a biplane area-length method, and were indexed to body surface area: maximum volume (before mitral valve opening), pre-A volume (before atrial contraction), and minimum volume (after atrial contraction).

LV mass was calculated by the Devereux formula and then indexed to body surface area [8].

Pulmonary artery systolic pressure (PASP) was estimated from peak tricuspid regurgitation jet velocities, adding right atrial pressure estimated from inferior vena cava diameter and respiratory changes [9].

All measurements were performed according to the recommendations of the European Association of Echocardiography/American Society of Echocardiography [10-12].

\section{Assessment of the LA function by 2D STE}

The global systolic LA myocardial strain was measured by 2D STE [13]. Gray scale image of apical 4-chamber views was obtained with the frame rates of $50-80 \mathrm{~Hz}$. Analysis was performed using QLAB advanced quantification software version 7.1 (Philips, The Netherlands). Automated tracking of myocardial speckles was reviewed and manually adjusted as minimally as possible. The cardiac cycle was demarcated by indicating QRS onset. Peak atrial longitudinal strain parameters were assessed as the average of 6 segmental 


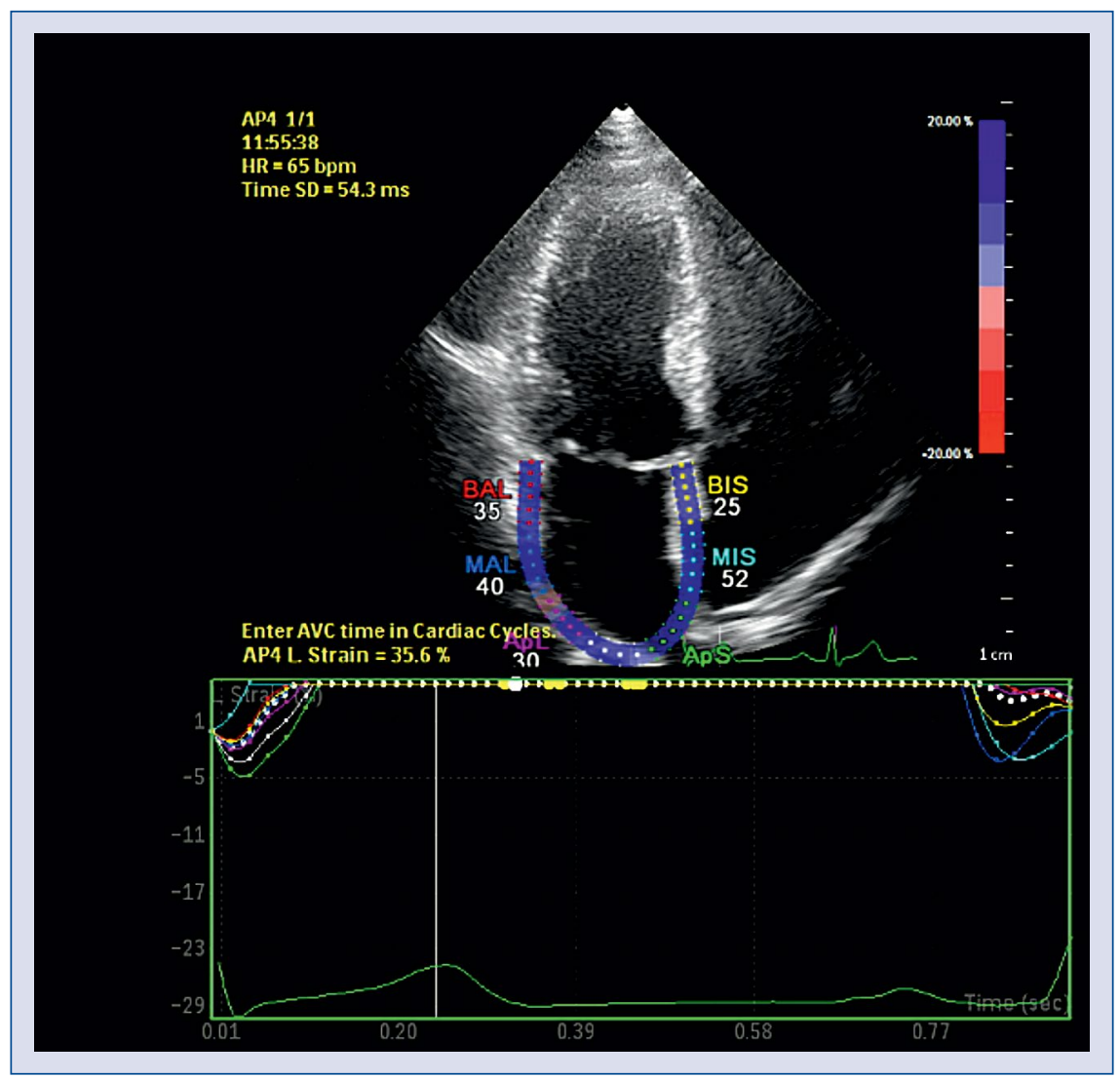

Figure 1. Measurement of peak left atrial longitudinal strain.

values in apical 4-chamber view demonstrated in Figure 1. In this study, to derive a noninvasive dimensionless parameter, the ratio of $\mathrm{E} / \mathrm{e}$ ' to LA peak strain was used to estimate the LA stiffness $[5,14,15]$.

\section{Statistical analyses}

The SPSS 16.0 statistical program (SPSS, Chicago, IL) was used for the statistical study. All values are given as mean and standard deviation. Values between different groups were compared using the independent-samples t-test. The $\chi^{2}$ test was used to assess differences between categorical variables. $\mathrm{P}<0.05$ was considered significant. The correlation between LA strain and stiffness and LA volume indices were evaluated using Pearson's correlation coefficient.

\section{Results}

There was no statistically significant difference between the patient group and the control group with regard to age, gender, heart rate, diameters of the LA, right atrium, LVEF, pulmonary artery systolic pressure, and body mass index (Table 1).
LA volumes were significantly larger in the ASA patients than in the normal control subjects (minimal volume index: $15.4 \pm 2.5$ vs. $10.7 \pm 1.6$, pre-A volume index: $22.3 \pm 2.8$ vs. $15.5 \pm 2.2 .5$, maximal volume index: $37.8 \pm 3.3$ vs. $26.3 \pm 2.9$, $\mathrm{p}<0.001$ for all) (Table 2).

ASA patients showed lower global LA strain $(25.3 \pm 5.2$ vs. $42.1 \pm 8.7, \mathrm{p}<0.001)$ and higher LA stiffness $(0.41 \pm 0.15$ vs. $0.14 \pm 0.05, \mathrm{p}<0.001)$ compared to normal control subjects (Table 2).

Although E/A ratio was not significantly different between the two groups, E, A values were higher, e', a' values were lower in the patient group. Additionally E/e' ratio was higher in the ASA patients $(10.0 \pm 2.6$ vs. $5.9 \pm 1.8, \mathrm{p}<0.001)$ (Table 1).

Figure 2 illustrated correlation between LA strain and LA volume indices. In the overall study population, the LA strain was strongly inversely correlated with LA minimal $(\mathrm{r}=-0.598, \mathrm{p}<0.001)$, pre-A $(\mathrm{r}=-0.603, \mathrm{p}<0.001)$, and maximal volume indices ( $\mathrm{r}=-0.680, \mathrm{p}<0.001)$ (Fig. 2).

Figure 3 illustrated correlation between LA stiffness and LA volume indices. In the overall study population, the LA stiffness and strain were 
Table 1. Comparison of clinical and conventional echocardiographic features of atrial septal aneurysm patients and controls group.

\begin{tabular}{|c|c|c|c|}
\hline & Patients $(n=34)$ & Controls $(\mathrm{n}=\mathbf{3 1})$ & $\mathbf{P}$ \\
\hline Age [years] & $44.2 \pm 12.3$ & $41.8 \pm 11.5$ & NS \\
\hline Male/female & $15 / 19$ & $14 / 16$ & NS \\
\hline Heart rate $[\mathrm{bpm}]$ & $61 \pm 8$ & $60 \pm 7$ & NS \\
\hline Body mass index $\left[\mathrm{kg} / \mathrm{m}^{2}\right]$ & $24 \pm 4.5$ & $23.5 \pm 3.0$ & NS \\
\hline Right atrium diameter [mm] & $35.5 \pm 3.6$ & $34.8 \pm 3.3$ & NS \\
\hline Left atrium diameter [mm] & $37.5 \pm 4.4$ & $36.2 \pm 3.6$ & NS \\
\hline Left ventricular ejection fraction [\%] & $62.2 \pm 5.1$ & $64.0 \pm 5.2$ & NS \\
\hline Systolic pulmonary artery pressure [mm Hg] & $25.8 \pm 5.6$ & $25.4 \pm 3.2$ & NS \\
\hline Left ventricular mass index $\left[\mathrm{g} / \mathrm{m}^{2}\right]$ & $85.1 \pm 15.5$ & $84.6 \pm 13.2$ & NS \\
\hline Mitral E [cm/s] & $81.1 \pm 16.8$ & $63.1 \pm 13.7$ & $<0.001$ \\
\hline Mitral A [cm/s] & $70.8 \pm 11.3$ & $58.8 \pm 9.2$ & $<0.001$ \\
\hline E/A ratio & $1.17 \pm 0.3$ & $1.1 \pm 0.28$ & NS \\
\hline $\mathrm{e}^{\prime}[\mathrm{cm} / \mathrm{s}]$ & $8.2 \pm 1.2$ & $10.9 \pm 1.7$ & $<0.001$ \\
\hline$a^{\prime}[\mathrm{cm} / \mathrm{s}]$ & $7.5 \pm 1.2$ & $8.5 \pm 1.4$ & 0.004 \\
\hline E/e' ratio & $10.0 \pm 2.6$ & $5.9 \pm 1.8$ & $<0.001$ \\
\hline
\end{tabular}

$\mathrm{E}$ - peak early diastolic filling velocity; A — peak late diastolic filling velocity; $\mathrm{e}^{\prime}$ - peak early diastolic velocity of the mitral annulus; $a^{\prime}$ - peak late diastolic velocity of the mitral annulus

Table 2. Comparison of left atrial (LA) volumes, stiffness and strain parameters of atrial septal aneurysm patients and controls group.

\begin{tabular}{lccc}
\hline & Patients $(\mathbf{n}=\mathbf{3 4})$ & Controls $(\mathbf{n}=\mathbf{3 1})$ & $\mathbf{P}$ \\
\hline LA minimal volume index $\left[\mathrm{mL} / \mathrm{m}^{2}\right]$ & $15.4 \pm 2.5$ & $10.7 \pm 1.6$ & $<0.001$ \\
LA pre-A volume index $\left[\mathrm{mL} / \mathrm{m}^{2}\right]$ & $22.3 \pm 2.8$ & $15.5 \pm 2.2$ & $<0.001$ \\
LA maximal volume index $\left[\mathrm{mL} / \mathrm{m}^{2}\right]$ & $37.8 \pm 3.3$ & $26.3 \pm 2.9$ & $<0.001$ \\
Global LA peak strain $[\%]$ & $25.3 \pm 5.2$ & $42.1 \pm 8.7$ & $<0.001$ \\
LA stiffness & $0.41 \pm 0.15$ & $0.14 \pm 0.05$ & $<0.001$ \\
\hline
\end{tabular}

strongly correlated with LA minimal ( $\mathrm{r}=0.495$, $\mathrm{p}<0.001)$, pre-A $(\mathrm{r}=0.595, \mathrm{p}<0.001)$, and maximal volume indices $(\mathrm{r}=0.625, \mathrm{p}<0.001)$ (Fig. 3).

\section{Discussion}

The main findings of our study are that patients with ASA showed a decreased global LA peak strain and an increased stiffness compared to the control subjects. LA strain and stiffness were strongly correlated with LA volume indices.

ASA is a risk factor for arterial embolism, as it frequently co-exists with ASD and PFO. Despite prior reports concerning paradoxical embolism through a PFO, recently suggested LA dysfunction and atrial arrhythmia, such as AF, might represent an alternate and additional mechanism for arterial embolism in these patients [15].
In previously published studies, the incidence of AF in ASA patients ranged from $0 \%$ to $23 \%$ [16, 17]. Janion et al. [2] reported that $\mathrm{p}$ wave dispersion and paroxysmal AF was higher in ASA patients.

In another study, it was reported that supraventricular arrhythmia and $\mathrm{p}$ wave dispersion was higher in ASA patients than in the control subjects [18].

Rigatelli et al. [3], in a study of 98 patients with ASA, found that LA dysfunction was higher in the patient groups.

We also demonstrated that impaired right and left atrial appendage function was higher in the ASA patients [19]. These studies, suggesting that the ASA may cause LA dysfunction.

Recently, LA myocardial strain has been confirmed as a reliable index that represents the magnitude of atrial deformation [20]. 




Figure 2. Correlation of left atrial strain and minimal (A), pre-A (B), and maximal (C) left atrial (LA) volume indices.
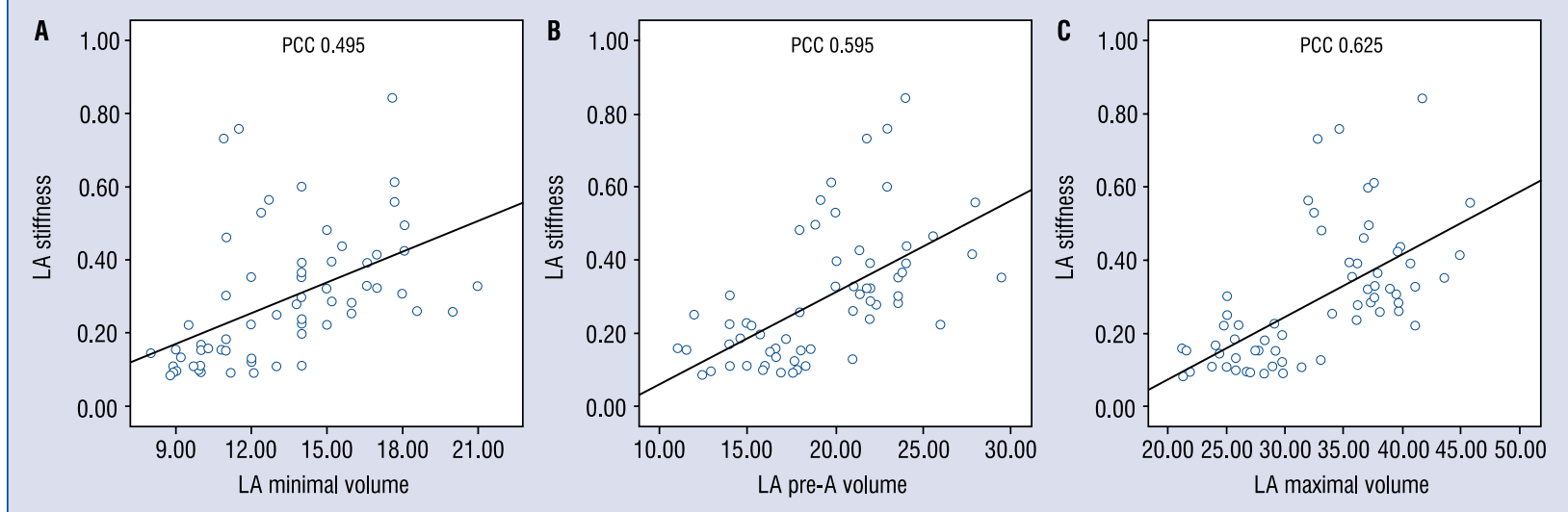

Figure 3. Correlation of left atrial stiffness and minimal (A), pre-A (B), and maximal (C) left atrial (LA) volume indices.

In previously published studies, global LA strain and strain rate were significantly reduced in patients with paroxysmal AF, compared with the normal control subjects [21,22].

Recently, Kurt et al. [14] suggested the noninvasive estimation of LA stiffness by using the E/e' ratio in conjunction with LA strain. In the study, noninvasively measured LA stiffness, as well as invasively measured LA stiffness, were increased in patients with diastolic heart failure. Also Yoon et al. [21] found that LA stiffness was significantly increased in patients with paroxysmal AF, and LA stiffness was correlated with LA volume indices.

In our study, LA strain was found to be lower in the patient group and it is believed that this may be related to atrial arrhythmia more common in those patients. Moreover, LA stiffness was higher in patients with ASA.

The most significant limitation of our study was the insufficient number of patients. Other limitations are that our study was not prospective and that the patients were not followed up for arrhythmias. Additionally, the degree and direction of protrusion was not considered.

\section{Conclusions}

In conclusion, this study demonstrated that LA strain was lower and stiffness was higher in ASA patients. These results are consistent with a few studies indicating an association between ASA and atrial dysfunction and paroxysmal AF.

The impaired strain and increased stiffness in the atrial myocardium may cause $\mathrm{AF}$ and arterial embolism in patients with ASA. It is clear that further comprehensive studies are needed regarding this issue. STE is a sensitive tool to assess impairment of LA mechanics, which is detectable in absence of changes in atrial size and volume, and may represent an early sign of atrial dysfunction in patients with ASA.

Conflict of interest: None declared 


\section{References}

1. Hanley PC, Tajik AJ, Hynes JK et al. Diagnosis and classification of atrial septal aneurysm by two-dimensional echocardiography: Report of 80 consecutive cases. J Am Coll Cardiol, 1985; 6: 1370-1382.

2. Janion M, Kurzawski J, Sielski J et al. Dispersion of P wave duration and $\mathrm{P}$ wave vector in patients with atrial septal aneurysm [published online ahead of print May 30, 2007]. Europace, 2007; 9: 471-474.

3. Rigatelli G, Aggio S, Cardaioli P et al. Left atrial dysfunction in patients with patent foramen ovale and atrial septal aneurysm: An alternative concurrent mechanism for arterial embolism? J Am Coll Cardiol Cardiovasc Interv, 2009; 2: 655-662.

4. Cameli M, Caputo M, Mondillo S et al. Feasibility and reference values of left atrial longitudinal strain imaging by two-dimensional speckle tracking. Cardiovasc Ultrasound, 2009, 7: 6 .

5. Kuppahally SS, Akoum N, Burgon NS et al. Left atrial strain and strain rate in patients with paroxysmal and persistent atrial fibrillation: relationship to left atrial structural remodeling detected by delayed-enhancement MRI. Circ Cardiovasc Imaging, 2010; 3 : 231-239.

6. D'Andrea A, Caso P, Romano S et al. Different effects of cardiac resynchronization therapy on left atrial function in patients with either idiopathic or ischaemic dilated cardiomyopathy: A two-dimensional speckle strain study. Eur Heart J, 2007; 28: 2738-2748.

7. Mugge A, Daniel WG, Angermann C et al. Atrial septal aneurysm in adult patients. A multicenter study using transthoracic and transesophageal echocardiography. Circulation, 1995; 91: 2785-2792.

8. Devereux RB, Reichek N. Echocardiographic determination of left ventricular mass in man. Anatomic validation of the method. Circulation, 1977; 55: 613-618.

9. Rudski LG, Lai WW, Afilal J et al. Guidelines for the echocardiographic assessment of the right heart in adults: a report from the American Society of Echocardiography endorsed by the European Association of Echocardiography, a registered branch of the European Society of Cardiology, and the Canadian Society of Echocardiography. J Am Soc Echocardiogr, 2010; 23: 685-713 (quiz 786-788).

10. Lang RM, Bierig M, Devereux RB et al. American Society of Echocardiography's Nomenclature and Standards Committee; Task Force on Chamber Quantification; American College of
Cardiology Echocardiography Committee; American Heart Association; European Association of Echocardiography; European Society of Cardiology: Recommendations for chamber quantification. Eur J Echocardiogr, 2006; 7:79-108.

11. Lancellotti P, Moura L, Pierard LA et al.; on behalf of the European Association of Echocardiography European Association of Echocardiography recommendations for the assessment of valvular regurgitation. Part 2: Mitral and tricuspid regurgitation (native valve disease). Eur J Echocardiogr; 2010; 11: 307-332.

12. Nagueh SF, Appleton CP, Gillebert TC et al.: Recommendations for the evaluation of left ventricular diastolic function by echocardiography. Eur J Echocardiogr; 2009; 10: 165-193.

13. Machino-Ohtsuka T, Seo $\mathrm{Y}$, Tada $\mathrm{H}$ et al. Left atrial stiffness relates to left ventricular diastolic dysfunction and recurrence after pulmonary vein isolation for atrial fibrillation. J Cardiovasc Electrophysiol, 2011; 22: 999-1006.

14. Kurt M, Wang J, Torre-Amione $\mathrm{G}$ et al. Left atrial function in diastolic heart failure. Circ Cardiovasc Imaging, 2009; 2: 10-15.

15. Demir M, Demir C. Assessment of atrial electromechanical coupling characteristics and P-wave dispersion in patients with atrial septal aneurysm. Southern Med J, 2011; 26: 549-557.

16. Janion M, Kurzawski J. Atrial fibrillation in patients with atrial septal aneurysm. Cardiol J, 2007; 14: 580-584.

17. Schneider B, Hofmann T, Meinertz T. Is there an association of atrial septal aneurysm with arrhythmias? Cardiology, 1999; 91: 87-91.

18. Deveci OS, Aytemir K, Okutucu S et al. Evaluation of the relationship between atrial septal aneurysm and cardiac arrhythmias via $\mathrm{P}$ wave dispersion and signal-averaged $\mathrm{P}$ wave duration. Ann Noninvasive Electrocardiol, 2010; 15: 157-164.

19. Demir M, Ozmen G, Keçoğlu S et al. Right and left atrial appendage function in patients with atrial septal aneurysm without patent foramen ovale. Acta Cardiol, 2012; 67: 457-460.

20. Di Salvo G, Caso P, Lo Piccolo R et al. Atrial myocardial deformation properties predict maintenance of sinus rhythm after external cardioversion of recent-onset lone atrial fibrillation: A color Doppler myocardial imaging and transthoracic and transesophageal echocardiographic study. Circulation, 2005; 112: 387-395.

21. Yoon YE, Kim HJ, Kim SA et al. Left atrial mechanical function and stiffness in patients with paroxysmal atrial fibrillation. J Cardiovasc Ultrasound, 2012; 20: 140-145.

22. Henein M, Zhao Y, Henein MY, Lindqvist P. Disturbed left atrial mechanical function in paroxysmal atrial fibrillation: A speckle tracking study. Int J Cardiol, 2012; 155: 437-441. 